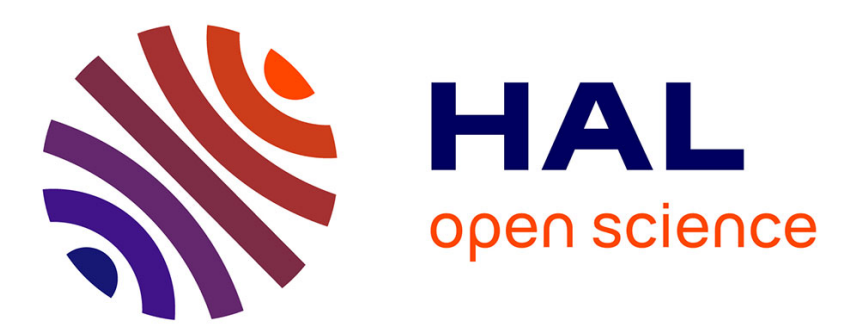

\title{
Assessment of exergy analysis of CFD simulations for the evaluation of aero-thermo-propulsive performance of aerial vehicles
}

\author{
Christelle Wervaecke, Ilias Petropoulos, Didier Bailly
}

\section{> To cite this version:}

Christelle Wervaecke, Ilias Petropoulos, Didier Bailly. Assessment of exergy analysis of CFD simulations for the evaluation of aero-thermo-propulsive performance of aerial vehicles. EUROGEN 2019, Sep 2019, GUIMARAES, Portugal. hal-02470727

\section{HAL Id: hal-02470727 \\ https://hal.science/hal-02470727}

Submitted on 7 Feb 2020

HAL is a multi-disciplinary open access archive for the deposit and dissemination of scientific research documents, whether they are published or not. The documents may come from teaching and research institutions in France or abroad, or from public or private research centers.
L'archive ouverte pluridisciplinaire HAL, est destinée au dépôt et à la diffusion de documents scientifiques de niveau recherche, publiés ou non, émanant des établissements d'enseignement et de recherche français ou étrangers, des laboratoires publics ou privés. 


\section{Assessment of exergy analysis of CFD simulations for the evaluation of aero-thermo-propulsive performance of aerial vehicles}

Christelle Wervaecke and Ilias Petropoulos and Didier Bailly

\section{Introduction}

Today more than ever, world energy resources seem limited and we have to carefully manage them. This is the obvious reason why future aerial transport concepts will be driven by energy efficiency criteria. This is both an economical and an ecological key issue. The exergy concept has been introduced so as to translate sources of entropy generation into power losses. Power, by definition, is the rate at which energy is consumed. Thus it is an essential information in the question of energetic systems optimization. Moreover, the exergy concept provides a common comparison metric which can be of great interest for multidisciplinary optimisation purposes.

Innovation and improvement require understanding. Nowadays Computation Fluid Dynamics (CFD) has become relevant to predict aerodynamic performances and the farfield drag decomposition method, proposed by Van Der Vooren and Destarac [1], has already provided valuable information about aerodynamical physical phenomena encountered in aircraft aerodynamics. Drag decomposition is now a common practice for aircraft design. Moreover farfield drag extraction approach is still a living research topic. For example, extensions for postprocessing unsteady flow fields have been proposed by Toubin et al. [2] and Gariepy et al.[3] and a new approach based on a Lamb vector approach is currently under study [4] [5]. However, future commercial aircraft are likely to get highly integrated propulsion

Christelle Wervaecke

ONERA Department of Aerodynamics, Aeroelasticity and Acoustics, The French Aerospace Lab, ChÃćtillon, F-92320, France, e-mail: christelle.wervaecke@onera.fr

Ilias Petropoulos

ONERA Department of Aerodynamics, Aeroelasticity and Acoustics, The French Aerospace Lab, Meudon, F-92190, France, e-mail: ilias.petropoulos@onera.fr

Didier Bailly

ONERA Department of Aerodynamics, Aeroelasticity and Acoustics, The French Aerospace Lab, Meudon, F-92190, France, e-mail: didier.bailly@onera.fr 
systems. Thereby, application of farfield drag methods will become difficult as those approaches require a clear separation between thrust and drag.

The need for a tool applicable to very innovative aircraft design or/and highlyintegrated propulsion systems along with the research of new methodologies enabling aerodynamic engineers to increase their understanding of physical flows have led the ONERA to develop a new postprocessing tool named FFX $[6,7,8]$ based on the exergetic approach. This tool is no more based on a mechanical balance as previous mentioned approaches but it is based on an energetic balance.

This paper gives attention to theoretical and numerical aspects of exergy analysis from solutions of the Reynolds-Averaged Navier-Stokes equations. Note that previous work has been presented at the AIAA AVIATION Forum by Petropoulos[9]. It was then a first attempt at analysing numerical behavior of our postprocessing tool and it proposed hints to find way to reduce numerical errors. The work presented in the current paper gives additional cases and analysis in order to deal with numerical error in depth and particularly to give a greater focus on 3D cases. It is organised as follows: the first part addresses the motivations for an exergy approach, the second part describes the exergy decomposition implemented in the FFX tool and the third part examines the relationship between CFD parameters such as simulation convergence or mesh refinement and the accuracy of the FFX decomposition in order to find ways to propose a more robust formulation.

\section{Why exergy?}

The exergy analysis has been proposed by Arntz [6] as an extension to the energy analysis method introduced by Drela [10]. It is an analysis which is conducted by the coupling of the first and second law of thermodynamics and that provides information about the energy amount that is theorically available. Whereas most previous field analysis methods have focused on forces information as drag and thrust, the exergy analysis focus on the idenfication and quantification of available useful work: sources, sinks and interaction. Thus it provides a very different kind of measurement that can enhance the physic flow comprehension of the aerodynamic engineer. As the formulation does not require any separation between drag and thrust, it can be applied even for high-integrated propulsive system.

To attain the climate global warming targets defined by ACARE (Advisory Council for Aeronautics Research in Europe)[11], there is a major focus from aircraft manufacturers to build more efficient aircraft. Aircraft manufacturers are urged to search for more efficient solutions and even disruptive technologies to reach the performance improvements required. All the subsystems which constitute the aircraft along with their mutual influence have to be taken into account in order to resolve these problems. The energy analysis method, derived from the first law of thermodynamic, is used in the design of aerospace systems. Fundamentally, an aircraft transforms chemical energy in other forms of energy. Kinetic, potential and mechanical work can be considered as conservative form of energy which can be 
converted into other forms without loss. However, heat, chemical and radiation energy cannot be completely converted in other forms of energy. For example, heat cannot be completely converted into work even from an idealised reversible cycle. So, exergy analysis consists in considering energy as the sum of two components: exergy and anergy. Exergy represents the available mechanical energy while anergy is the part of energy which cannot be transformed due to irreversible processes.

Furthermore, the exergy approach is based on a balance equation derived from the first and the second law of thermodynamics. It is possible to locally evaluate not only the losses associated with each physical phenomenon in the considered system, but also those associated with phenomena occurring outside it and that can be considered as a measure of waste. Overall system improvement can be achieved by reducing losses from internal irreversibilities and generally the total waste of the system. So exergy appears to be well adapted to improve complex systems where different energy transformations take place. This methodology enables to study a complete aircraft as being constituted of subsystems of different nature by using a common metric.

\section{Formulation}

In a nutshell, exergy is the energy that is available and that can be transformed to a useful form of energy. By combining the first and the second laws of thermodynamics, it handles energy and entropy together and can be defined as:

$$
\mathcal{E}=\Delta h_{i}-T \Delta s
$$

where the term $h_{i}$ denotes specific total enthalpy, $\Delta h_{i}=\left(h_{i}-h_{i, \infty}\right)$ and the subscript $\infty$ indicates reference conditions which are usually taken as the atmosphere freestream flow. The term $s$ denotes entropy and $\Delta s=\left(s-s_{\infty}\right)$. FFX provides the exergy-based formulation summarized within the equation (2) for aeropropulsive performance assessment developed by A. Arntz during his $\mathrm{PhD}$ thesis [6]. More details of the theoretical development are available in reference [8]. The general exergy-based formulation can be written as:

$$
\dot{\mathcal{E}}_{\text {prop }}+\dot{\mathcal{E}}_{q}=W \dot{\Gamma}+\dot{\mathcal{E}}_{m}+\dot{\mathcal{E}}_{t h}+\dot{A}_{t o t}
$$

Each term of this exergy decomposition will be described more specifically hereafter. Figure (1) provides a scheme depicting notations in FFX's framework. It should be noted that the method aims at evaluating the time-averaged changes in exergy hence the introduction of exergy rate terms denoted by $\dot{\mathcal{E}}$. The destroyed exergy has been called anergy and the rate of anergy, i.e. the unusable part of energy, is denoted by $\dot{A}$. 


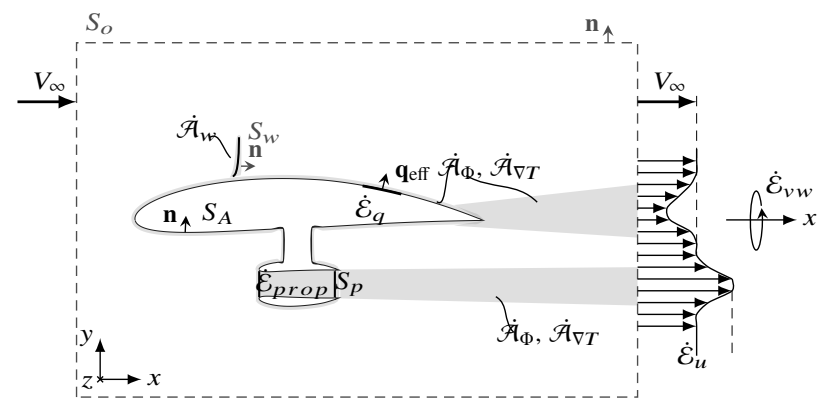

Fig. 1: Notations of the exergy decomposition.

The left hand side of equation (2) represents the total exergy supplied to the flow by the propulsion system or by heat conduction. The first term $\dot{\mathcal{E}}_{\text {prop }}$ is defined as follows:

$$
\dot{\mathcal{E}}_{\text {prop }}=\int_{S_{p}}-\rho \delta h_{i} \mathbf{V} \cdot \mathbf{n} d S+T_{\infty} \int_{S_{p}} \rho \delta s \mathbf{V} \cdot \mathbf{n} d S
$$

It is the rate of exergy supplied by the propulsion system and $S_{p}$ is the surface delimiting it. The first part is the power supplied to the flow while the second one represents thermodynamic losses that have occurred within the propulsion system. The other term of supplied exergy, $\dot{\mathcal{E}}_{q}$ is written as:

$$
\dot{\mathcal{E}}_{q}=\int_{S_{A}}-\mathbf{q}_{e f f} \cdot \mathbf{n} d S+\int_{S_{A}} \frac{T_{\infty}}{T} \mathbf{q}_{e f f} \cdot \mathbf{n} d S
$$

It corresponds to the rate of exergy transfer by heat conduction through the surface of the airplane. The first term is the heat transferred by conduction and the second one is the associated anergy. Both of these integrals are non zero only on non-adiabatic surfaces.

The right hand side of equation (2) corresponds to the sum of exergy consumed by the airplane, exergy remaining in the flow and exergy destroyed through irreversible processes (which is anergy). First, the term $W \dot{\Gamma}$ is defined as follows:

$$
W \dot{\Gamma}=\int_{S_{o}} \rho\left(u-V_{\infty}\right) V_{\infty} \mathbf{V} \cdot \mathbf{n}+\left(p-p_{\infty}\right) \mathbf{V}_{\infty} \cdot \mathbf{n} d S
$$

It represents the mechanical exergy part consumed by the airplane. As the exergy balance equation is written assuming a steady flow, the term $W \dot{\Gamma}$ represents the energy consumed to maintain a steady path: whether cruise, climb or descent. Considering an unpropelled configuration $\left(\dot{\mathcal{E}}_{\text {prop }}\right.$ and $\dot{\mathcal{E}}_{q}$ are both zero), $W \dot{\Gamma}$ matches with the corresponding drag coefficient when choosing a suitable nondimensionalization. It should be underlined that the above expression provides no distinction between thrust and drag. 
Among sources of exergy still available in the flow, the term $\dot{\mathcal{E}}_{m}$ represents the rate of mechanical exergy:

$$
\dot{\mathcal{E}}_{m}=\dot{\mathcal{E}}_{u}+\dot{\mathcal{E}}_{v w}+\dot{\mathcal{E}}_{p}
$$

where

$$
\begin{aligned}
\dot{\mathcal{E}}_{u} & =\int_{S_{o}} \frac{1}{2} \rho u^{2} \mathbf{V} \cdot \mathbf{n} d S \\
\dot{\mathcal{E}}_{v w} & =\int_{S_{o}} \frac{1}{2} \rho\left(v^{2}+w^{2}\right) \mathbf{V} \cdot \mathbf{n} d S \\
\dot{\mathcal{E}}_{p} & =\int_{S_{o}}\left(p-p_{\infty}\right)\left(\mathbf{V}-\mathbf{V}_{\infty}\right) \cdot \mathbf{n} d S
\end{aligned}
$$

It is the sum of the streamwise kinetic energy $\left(\dot{\mathcal{E}}_{u}\right)$, the transverse kinetic energy deposition $\left(\dot{\mathcal{E}}_{v w}\right)$ and a third term described as the exterior pressure-work $\left(\dot{\mathcal{E}}_{p}\right)$. The other term of available exergy is the term $\dot{\mathcal{E}}_{t h}$ which is the rate of thermal exergy:

$$
\begin{aligned}
\dot{\mathcal{E}}_{t h}=\int_{S_{o}} \rho \delta e \mathbf{V} \cdot \mathbf{n} d S & +\int_{S_{o}} p_{\infty} \mathbf{V} \cdot \mathbf{n} d S \\
& -T_{\infty} \int_{S_{o}} \rho \delta s \mathbf{V} \cdot \mathbf{n} d S
\end{aligned}
$$

Finally, the term $\dot{A}_{t o t}$ denotes the rate of anergy generation, or equivalently of exergy destruction, by irreversible phenomena which are viscous dissipation (first term), thermal conduction (second term) and shockwaves (third term):

$$
\dot{A}_{t o t}=\dot{A}_{\Phi}+\dot{A}_{\nabla T}+\dot{A}_{w}
$$

where

$$
\begin{aligned}
\dot{A}_{\Phi} & =\int_{v} \frac{T_{\infty}}{T} \Phi_{e f f} d v \\
\dot{A}_{\nabla T} & =\int_{v} \frac{T_{\infty}}{T^{2}} K_{e f f}(\nabla T)^{2} d v \\
\dot{A}_{w} & =T_{\infty} \int_{S_{w}} \rho \delta s \mathbf{V} \cdot \mathbf{n} d S
\end{aligned}
$$

Note that all terms of the decomposition (2) are nondimensionalized by the coefficient $: \frac{1}{2} \rho_{\infty} \mathbf{V}_{\infty}{ }^{3} S_{\text {ref }}$. The term $S_{\text {ref }}$ denotes a reference surface. 


\section{Accuracy assessment}

\subsection{Implementation}

The exergy decomposition has been implemented in ONERA's in-house postprocessing tool named FFX. The implementation is strongly coupled with the Cassiopée library [12]. It benefits of its Python/C++ environment and can handle both structured and unstructured meshes along with cell-centered or vertex-centered solutions. As the exergy formulation is still under study, it is necessary to maintain a very flexible implementation framework such as to be able to test and modify rapidly some terms if required. The Cassiopée platform is appropriate for such investigations. Moreover, the FFX tool has already been applied by some of ONERA's industrial partners on complex configurations see Tailliez [13], Couilleaux [14] and Wiart [15]. The first results seem quite encouraging.

\subsection{Sensitivity analysis}

This section deals with academic applications investigated in order to assess the FFX tool accuracy. All RANS computations were performed with the elsA solver of ONERA [16].

\subsubsection{NACA0012 case}

In order to assess the sensitivity of the exergy decomposition to mesh refinement and CFD computation convergence, this section focuses on a 2D academic test case: the flow around a NACA0012 airfoil. There is a lot of information for this test case as it was used as a verification test case for Drag Prediction Workshop 5 (DPW5) and Drag Prediction Workshop 6 (DPW-6)[17]. A sequence of nested grids are provided on the NASA Turbulence Modeling Resource website [18], the number of cells ranging from 3500 to $14.710^{6}$ and each coarser grid is exactly composed of every-other-point of the next finer grid. The grids have a farfield extent of about $500 \mathrm{c}$. The reference state conditions are Mach number $\mathrm{M}=0.15$, the Reynolds number per chord length is $\operatorname{Re}=6$ million and the angle of attack is $\alpha=10^{\circ}$. Figure (2) shows the Mach number field around the NACA0012 airfoil and table (1) gives the number of cells for each grid considered in this study.

Although the flow around a $2 \mathrm{D}$ profile is a very simple case, it provides a very good frame to assess a postprocessing tool accuracy as CFD convergence can be reached (indeed the more complex the case is, the more touchy the convergence becomes) and mesh refinement is easily achieved. Moreover, a deeper understanding of the exergetic decomposition terms is much easier for such a case and gives insight into its physical meanings. As we consider an unpowered configuration, the terms 


\begin{tabular}{|c|c|c|c|c|c|c|c|}
\hline Mesh & 1 & 2 & 3 & 4 & 5 & 6 & 7 \\
\hline Ncell & 3584 & 14336 & 57344 & 229376 & 917504 & 3670016 & 14680064 \\
\hline
\end{tabular}

Table 1: NACA0012 - Size of the 7 nested structured grids.

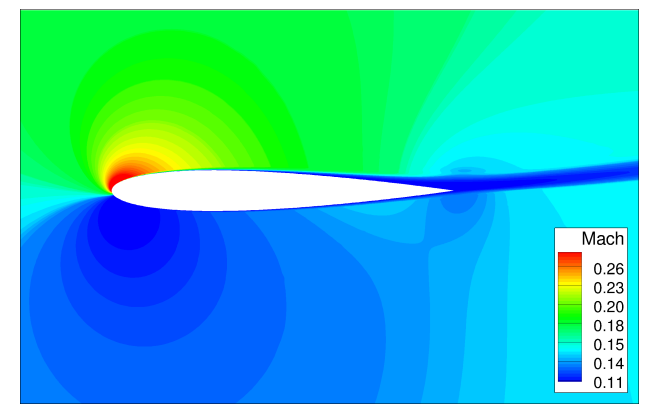

Fig. 2: Mach number field around the NACA0012 airfoil.

$\dot{\mathcal{E}}_{\text {prop }}$ and $\dot{\mathcal{E}}_{q}$ of the balance equation (2) are zero. The term $W \dot{\Gamma}$, appropriately nondimensionalized, matches the total drag coefficient. Finally, the amount of exergy still available within the flow is composed of the mechanical and the thermal exergy. These terms decrease in the wake as the amount of anergy, generated by viscous and thermal phenomena (no shockwave is formed at these conditions), increases. Figure (3) shows the evolution of the exergy decomposition's terms with respect to the location of the downstream limit of the control volume (driven by the $x_{T P}$ parameter) for mesh 5. There is a good agreement between the term $W \dot{\Gamma}$ and the farfield drag coefficient $c d_{f f}$, evaluated by the ONERA far-field drag code, and exergy and anergy terms behave as expected (note that it takes a very long distance for the mechanical exergy to vanish). The rate of mechanical exergy decreases towards zero as the Trefftz plane moves downstream of the body. Its decomposition shows that after some chords the total mechanical exergy reduces to the streamwise kinetic energy of the wake. In the same way, the rate of thermal exergy decreases towards zero. These behaviors are due to irreversible process occurring in the wake (dissipation). These exergy losses are counterbalanced by the increase of the total anergy term.

In the first part of this NACA0012 case analysis, the exergetic balance is estimated several times as the residuals of the CFD computation decrease. The aim is to analyse whether some terms are more sensitive to CFD convergence and which level of convergence is required to attain a good exergetic decomposition accuracy. The downstream limit of the control volume is set up at $x_{T P}=2$ for the following results. The figures (4) and (5) provide some results for mesh 3 and mesh 5 (see table (1)). The relative error is estimated as the difference between final value, i.e. when CFD convergence is achieved, and current value for each term of the formulation (in percentage). The absolute value of each term is also given so as to indicate 


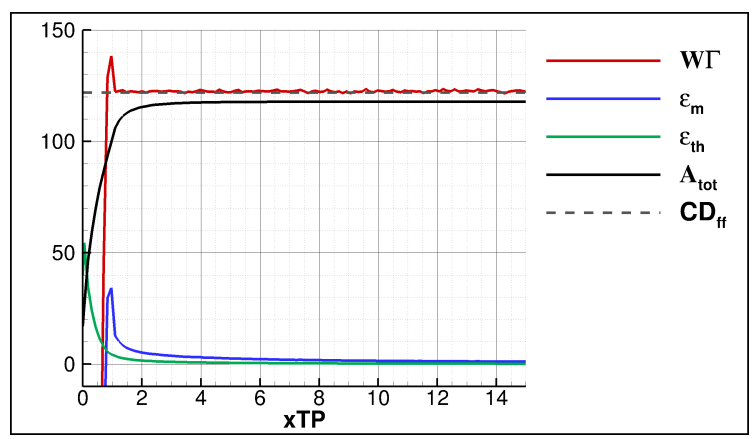

Fig. 3: NACA0012 - Mesh 5. Evolution of the exergy decomposition's terms with respect to the location of the downstream limit of the control volume.

their order of magnitude: thermal and mechanical exergy are smaller in absolute value than the other terms. Figure (6a) gives the residual for the equation of mass conservation with respect to the number of iterations. It shows that a residual around $\sim 10^{-4}, \sim 10^{-5}$ are quickly reached. That is why it is the starting point for plots on exergy relative errors. In figure (4) and figure (5), nearfield $\left(c d_{n f}\right)$ and farfield $\left(c d_{f f}\right)$ drag coefficients are also given. They have been computed by the ONERA in-house FFD (Far Field Drag) tool. It enables to compare exergy balance and drag evaluation requirements on CFD convergence. When looking for a relative error of $1 \%$, the total anergy term $\dot{A}_{t o t}$ behaves as well as $c d_{f f}$ or $c d_{n f}$ on both meshes and even reached this threshold faster than these terms on the coarsest mesh (mesh 3). Smaller levels of relative error are more quickly reached by $c d_{f f}$ and $c d_{n f}$ afterward. It is not obvious from results of mesh 3 but for all finer meshes, the thermal exergy term $\dot{\mathcal{E}}_{t h}$ converges faster than the other terms whereas the mechanical exergy term $\dot{\mathcal{E}}_{m}$ converges a little bit slower. It should be kept in mind that a relative error of $1 \%$ means a difference of $5.310^{-2} p c$ (pc means power count where power count is, by analogy with drag count, equal to a dimensionless exergy coefficient of 0.0001 ) for the mechanical exergy term and $1.610^{-2} p c$ for the thermal exergy term whereas it implies a difference of $1.1 p c$ for the total anergy term or for the term $W \dot{\Gamma}$. Plots have to be interpreted cautiously.

The other interesting part of this study is the grid convergence analysis. Are all terms equally affected by mesh refinement or are there terms that can be estimated with good confidence even on a coarse mesh? Figure (7) shows values of the FFX decomposition with respect to mesh refinement. Plots have been split to give more clarity in the two different scales: $W \dot{\Gamma}$ and $\dot{A}_{t o t}$ on one hand, $\dot{\mathcal{E}}_{m}$ and $\dot{\mathcal{E}}_{t h}$ on the other hand. While the two first meshes are quite too coarse to provide reliable values, specifically for the $W \dot{\Gamma}$ term, mesh 3 and finer meshes give reasonable accuracy. They are within 1 counts of the finest mesh value for the $W \dot{\Gamma}$ value. Although a theoretically exact exergy decomposition is not available for this case, mesh 7 is considered fine enough (with $14,7 \mathrm{M}$ of cells) to provide reference values. Figure (6b) gives the relative differences between the current values and these reference 

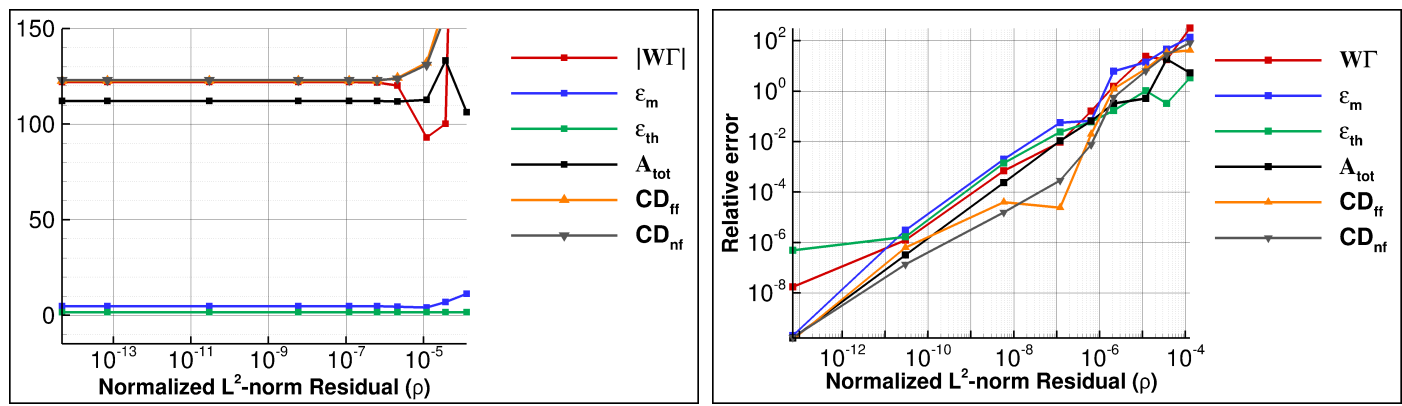

(a) Values of the FFX decomposition with respect to CFD(b) Relative error in FFX's terms with respect to CFD conconvergence vergence

Fig. 4: NACA0012 - Results on mesh 3.

values (in percentage) for each mesh. The reduction of relative error seems to behave almost linearly with the number of cells.

The analysis of these cases shows that there is no great difference between far field drag terms behaviour and exergetic terms behaviour when dealing with CFD convergences. As ONERA's far field drag approach has been studied for more than fifteen years and has been proved to be quite effective, these first results then give confidence in the exergetic approach.
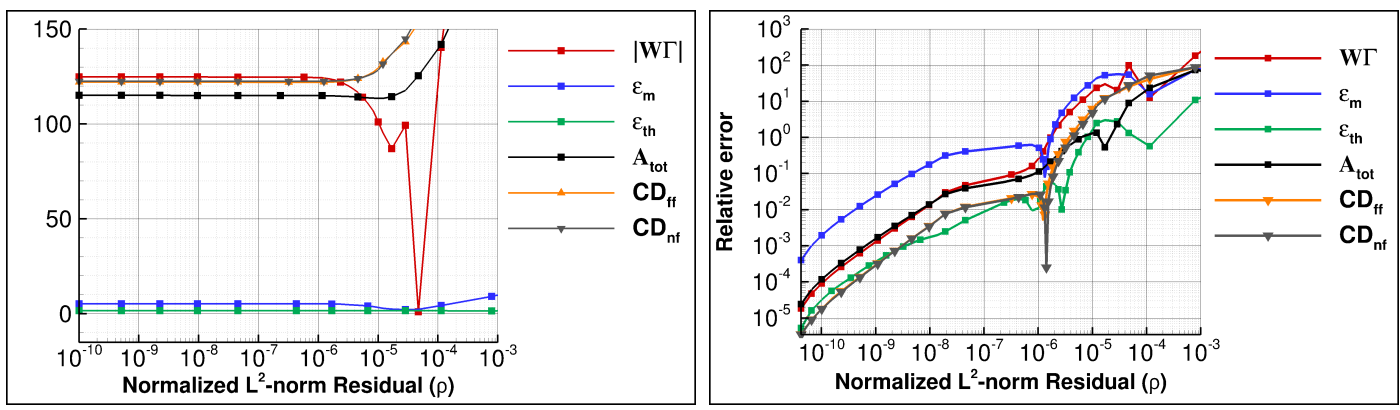

(a) Values of the FFX decomposition with respect to CFD(b) Relative error in FFX's terms with respect to CFD conconvergence vergence

Fig. 5: NACA0012 -Results on mesh 5. 


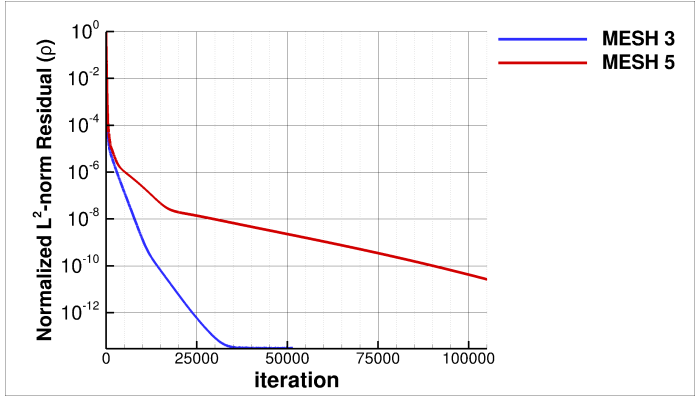

(a) Convergence of the CFD computation for mesh 3 and mesh 5 .

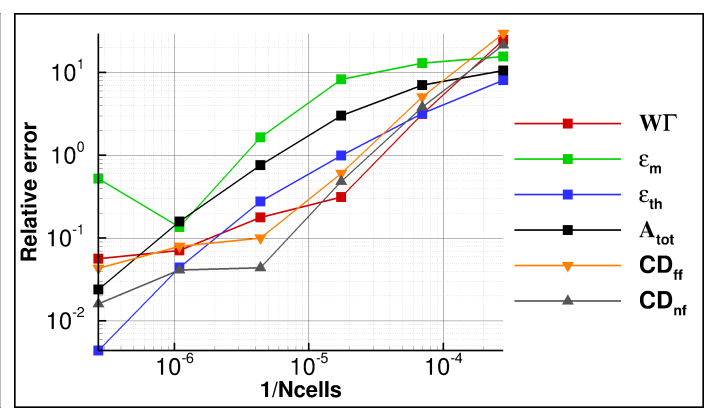

(b) Numerical error with respect to mesh refinement.

Fig. 6: NACA0012
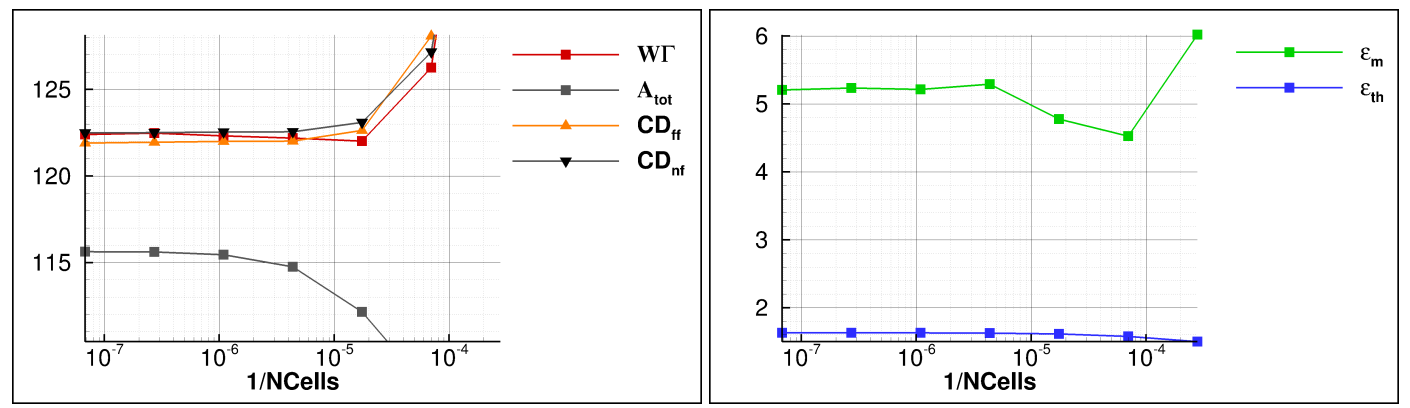

Fig. 7: NACA0012 - Values of the FFX's terms with respect to mesh refinement.

\subsubsection{NASA-CRM case}

As it has been widely experimentally and numerically studied, the wing-body configuration of the Common Research Model (CRM) is a good case to assess the accuracy and sensibility of the FFX post-processing tool. Exergy decomposition has already been studied on this configuration during the early development of the formulation in Arntz[19]. At that time, the post-processing FFX tool was a prototype implemented in a FORTRAN code. Nowadays, it is a Python/C++ tool which is more mature and does not introduce any correction to account for spurious exergy. What is called spurious exergy is exergy having no physical meaning and only generated by numerical errors. Instead, it has been decided to investigate more carefully this term in order to get a better understanding. Figure (8a) gives an overview of this configuration. The freestream aerodynamic conditions are Mach number of $M=0.85, C L=0.5$ and Reynolds number $R e=510^{6}$. Note that it is a transonic case which introduces a shock wave phenomenon in addition to $3 \mathrm{D}$ effects compared to the previous subsonic NACA0012 case. Several meshes are available for this configuration at ONERA and 
CFD computations and drag analyses have been documented in Hue[20]. These meshes, carefully generated in the context of the Drag Prediction Workshop, have good qualities in term of grid spacing, stretching ratio and grid orthogonality. Note that they have been built in order to meet the drag prediction requirements. It is far from sure that they are also suitable for exergy evaluation. This is a question that our study aims at addressing: what is a good quality mesh from an exergy analysis point of view? Finally they belong to a family of six grids built by coarsening the finest one, which is a good point for mesh convergence analysis. In order to guarantee a CFD convergence down to machine precision, computations has been carried on for a great number of iterations. Figure (8b) shows the residual of the mass conversation equation with respect to the number of iterations and confirms that machine precision is achieved.

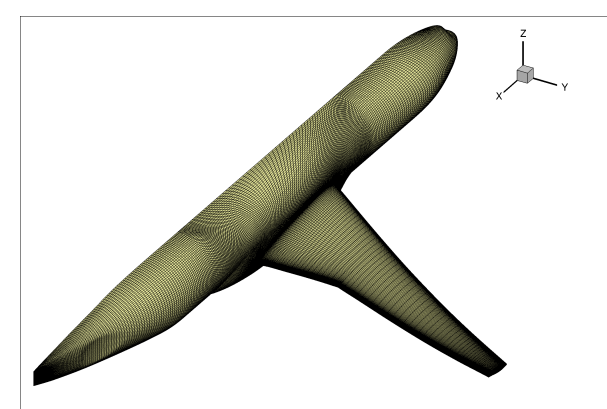

(a) Overview of the CRM-NASA configuration

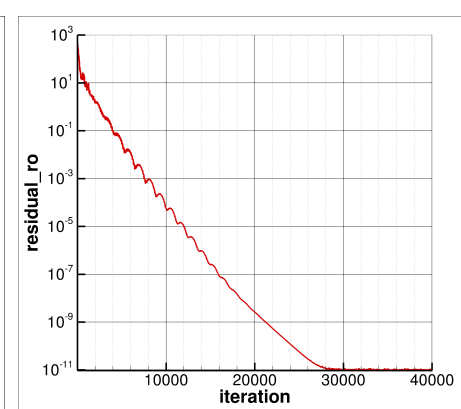

(b) Residual on the mass conservation equation with respect to iteration numbers

Fig. 8: CRM case

Table (2) gives the value of the FFX's components for four meshes composed of $2 \mathrm{M}, 5 \mathrm{M}, 17 \mathrm{M}$ and $41 \mathrm{M}$ of cells. The thermal exergy part is quite well predicted for every mesh (relative error is less than 5.3\% ) but it should be noted that this term takes small values. Good accuracy is also obtained for the mechanical exergy part with less than $2.6 \%$ relative error even for the coarsest mesh. The amount of total anergy is less accurately predicted on the coarsest mesh (7.1\% of relative error) but this error decreases as the mesh get finer and is less than $2 \%$ on L4. Finally the term $W \dot{\Gamma}$ still seems to be the most difficult to estimate accurately as the error is higher on L3 than on L2 and reaches 10\%. It is consistent with observations on the NACA0012 case.

As for the NACA0012 test case, the accuracy of the FFX decomposition with respect to CFD convergence has been studied on meshes L2 to L4. The figure (9) only shows the result for mesh L3 but the terms have a very similar behaviour on all three meshes. The total anergy term provides the fastest convergence and is even faster than the far-field drag term. The mechanical exergy, which is far from being 


\begin{tabular}{|c|c|c|c|c|}
\hline CRM & L'2 & L'3 & L'4 & L'5 \\
\hline Ncells & 2156544 & 5111808 & 17252352 & 40894464 \\
\hline$W \dot{\Gamma}$ & 257.97 & 256.18 & 254.63 & 254.35 \\
\hline$\dot{\mathcal{E}}_{m}$ & 87.86 & 89.14 & 90.77 & 91.85 \\
\hline$\dot{\mathcal{E}}_{\text {th }}$ & 1.84 & 1.81 & 1.86 & 1.87 \\
\hline$\dot{A}_{\text {tot }}$ & 139.05 & 143.10 & 147.04 & 149.40 \\
\hline
\end{tabular}

Table 2: Contributions to the exergetic balance for different meshes.

a negligible term for this case, shows a very good convergence too. As previously explained, the thermal exergy part takes too small values to be able really to appraise its convergence performance. Finally the $W \dot{\Gamma}$ is more demanding in terms of CFD convergence in order to achieve the same precision as the other terms.
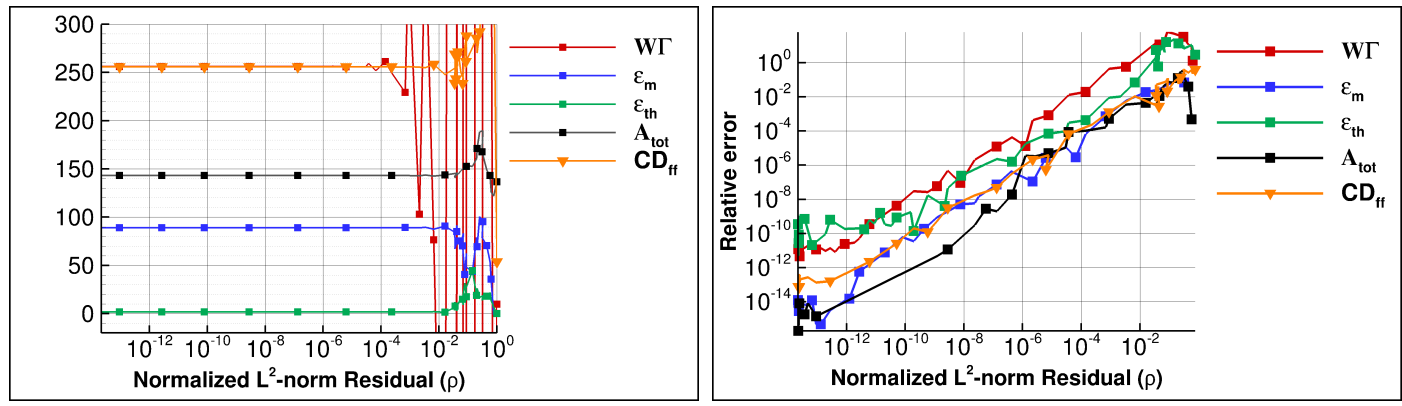

(a) Values of the FFX decomposition with respect to CFD(b) Relative error in FFX's terms with respect to CFD conconvergence vergence

Fig. 9: CRM - Results on mesh LP3.

Another aspect that could help improving the post-processing accuracy is the definition of the control volume, where the exergy balance is calculated. Destarac[21] and al. have carefully designed control volumes for the far-field drag method. The objective is to exclude any volume where no physical phenomenon occurs and only spurious drag can be generated. The figure (10) gives illustration of the three definitions of control volume considered in this study. It should be reminded that a single FFX balance is achieved for a fixed downstream Trefftz plane (defined by the $x_{T P}$ parameter). All other boundaries of the control volume can be freely defined as long as they are far enough for the flow to get back to thermodynamic equilibrium. The first volume $(V 1)$ extends up to the farfield boundary condition in all directions (except for the $x_{T P}$ plane as explained). The table (3) gives FFX terms for the three volumes. The second volume $(V 2)$ is defined by limiting the control volume in $\mathrm{x}, \mathrm{y}$ and $\mathrm{z}$ direction as $x_{\min }=z_{\min }=-50$ and $y_{\max }=z_{\max }=50$. There are very few 
differences in the results between $V 1$ and $V 2$ and even negligible differences (values are absolutely the same up to 2 decimal digits) for the anergy terms. It means that, for this case, there is not a significant amount of spurious anergy generated outside this box. Finally the volume $V 3$ is a volume similar to one of the volumes used for far-field drag analysis. It is built as a viscous volume defined by physical sensors, plus a shock volume also identified thanks to physical sensors. Whereas viscous and thermal anergy ( $\dot{A}_{\Phi}$ and $\dot{A}_{\nabla T}$ ) still have quite the same values for $V 3$, mechanical and thermal exergy are badly predicted. This is a direct result of thermodynamic equilibrium not being is not yet achieved on the boundaries on this control volume.

\begin{tabular}{|c|c|c|c|c|c|}
\hline $\begin{array}{c}\text { Control } \\
\text { volume }\end{array}$ & $W \dot{\Gamma}$ & $\dot{\mathcal{E}}_{m}$ & $\dot{\mathcal{E}}_{t h}$ & $\dot{A}_{\Phi}$ & $\dot{A}_{\nabla T}$ \\
\hline V1 & 257.97 & 87.86 & 1.84 & 122.72 & 11.96 \\
\hline V2 & 258.78 & 88.25 & 1.60 & 122.72 & 11.96 \\
\hline V3 & 257.82 & 466.73 & -375.30 & 122.70 & 11.96 \\
\hline
\end{tabular}

Table 3: FFX balance at $x_{T P}=90$ for 3 control volumes: V1 is the classical FFX control volume, $\mathrm{V} 2$ is the control volume with restriction in all directions and V3 is the FFD-like control volume.

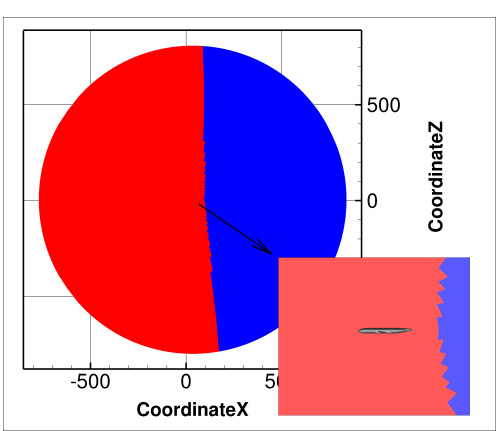

(a) Classical control volume without restriction (V1)

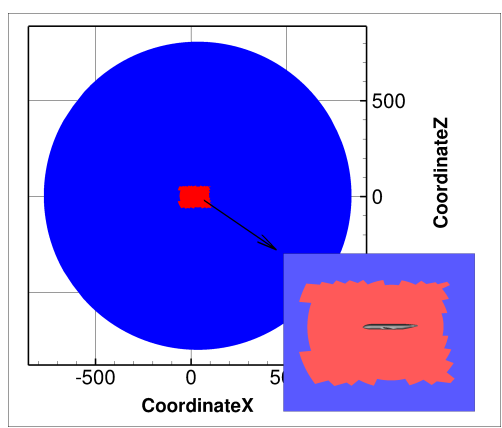

(b) Restriction in all 3 directions (V2)

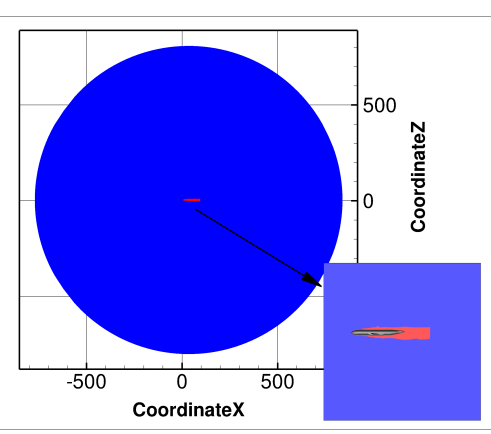

(c) FFD-like control volume (V3)

Fig. 10: Control volumes for the CRM L2 case. The control volume is marked by the red contour region.

To sum up, the convergence of the term $W \dot{\Gamma}$ is the trickiest one as it has been also confirmed for other test cases (not presented here). Moreover, the evaluation of the anergy terms suffers with greater error as it deals with gradients computation and integration in a larger domain. 


\section{Conclusion}

A new promising post-processing method has been presented based on the exergy concept. Such an analysis can be used as a basis for the construction of objective functions for optimization processes. Although it has already been widely studied from a physical point of view by Arntz[6], there was still a need for an improved numerical understanding. Indeed, as long as numerical methods are concerned, discretization of the continuous flow field, truncation error, convergence of iterative processes imply that the result can not be free from error. So the aim of this paper was to evaluate the loss of accuracy associated with mesh discretization and imperfect CFD converged solutions and to find strategies to minimize it. Of course each flow simulation and analysis will have its own distinctive characteristics and no conclusion can be drawn which would be universal. However it has been found that the term $W \dot{\Gamma}$ was the most sensitive and it will be of great interest to look further into this issue. Comparisons with the farfield approach prove that, in term of mesh convergence and sensitivity to CFD convergence, the exergy decomposition's terms show a quite equivalent behaviour. Finally some attempts at increasing the postprocessing accuracy have been achieved through a reduction of the control volume. Although no huge differences are obtained for the CRM case presented in this paper, it seems wise to exclude from the control volume region where the mesh is too coarse to accurately predict the flow. Indeed these are locations where spurious anergy will be produced. So the control volume has to be large enough for the flow to return to thermodynamic equilibrium but small enough to exclude zones where the mesh become too coarse. Some additional work will be carried on to automatically define a control volume that meets this requirement.

\section{References}

1. Van der Vooren, J. and Destarac, D. Drag/Thrust Analysis of a Jet-propelled Transonic Transport Aircraft: Definition of Physical Drag Components. Aerospace Science and Technology 8 , 545-556 (2004).

2. Toubin, H. and Bailly, D. Development and Application of a New Unsteady Far-Field Drag Decomposition Method. AIAA Journal 53, No. 11, 3414-3429. (2015).

3. Gariépy, M., Trépanier, J.-Y., and Malouin, B. Generalization of the Far-Field Drag Decomposition Method to Unsteady Flows. AIAA Journal 51, No. 6, 1309-1319. (June 2013).

4. Mele, B. and Tognaccini, R. Aerodynamic force by Lamb vector integrals in compressible flow. Physics of Fluids 26, 1-16 (May 2014).

5. Wu, J., Liu, L., and Liu, T. Fundamental theories of aerodynamic force in viscous and compressible complex flows. Progress in Aerospace Sciences 99, 27-63 (May 2018).

6. Arntz, A. Civil Aircraft Aero-thermo-propulsive Performance Assessment by an Exergy Analysis of High-fidelity CFD-RANS Flow Solutions. Ph.D. Dissertation, Lille 1 Université Sciences et Technologies, Lille, France, (2014).

7. Arntz, A., Atinault, O., Destarac, D., and Merlen, A. Exergy-based Aircraft Aeropropulsive Performance Assessment: CFD Application to Boundary Layer Ingestion. 32nd AIAA Applied Aerodynamics Conference, AIAA AVIATION Forum, AIAA paper 2573 (2014). 
8. Arntz, A., Atinault, O., and Merlen, A. Exergy-Based Formulation for Aircraft Aeropropulsive Performance Assessment: Theoretical Development. AIAA Journal 53, No. 6 (2015).

9. Petropoulos, I., Wervaecke, C., D., B., and T., D. Numerical investigations of the exergy balance method for aerodynamic performance evaluation. AIAA AVIATION Forum (17-21 June 2019, Dallas, Texas).

10. Drela, M. Power Balance in Aerodynamic Flows. AIAA Journal 47, No. 7, 1761-1771 (July 2009).

11. ACARE - FlightPath 2050 Goals. https://www.acare4europe.org/sria/ flightpath-2050-goals.

12. Benoit, C., Péron, S., and Landier, S. Cassiopee: a CFD pre- and post-processing tool. Aerospace Science and Technology 45, 272-283 (2015).

13. Tailliez, C. and Arntz, A. CFD assessment of the use of exergy analysis for losses identification in turmbomachines flows. 53rd 3AF International Conference on Applied Aerodynamics, Salon de Provence, France (March 2018).

14. Couilleaux, A. and Arntz, A. Exergy analysis for a CFD-based turbofan exhaust mixer performance improvement. 53rd 3AF International Conference on Applied Aerodynamics, Salon de Provence, France (March 2018).

15. Wiart, L. and Negulescu, C. Exploration of the Airbus âĂIJNAUTILIUSâĂİ engine integration concept. 31st Congress of the International Council of the Aeronautical Sciences, Belo Horizonte, Brazil (September 2018).

16. Cambier, L., Heib, S., and Plot, S. The Onera elsA CFD software: input from research and feedback from industry. Mechanics \& Industry 14, No. 3, 159-174 (2013).

17. Drag Prediction Workshop website. https://aiaa-dpw.larc.nasa.gov/.

18. NASA Turbulence Modeling Resource website. https://aiaa-dpw.larc.nasa.gov/.

19. Arntz, A. and Hue, D. Exergy-Based Performance Assessment of the NASA Common Research Model. AIAA Journal, 54, No. 1, 88-100 (2016).

20. Hue, D., Chanzy, Q., and Landier, S. DPW-6: Drag Analyses and Increments Using Different Geometries of the Common Research Model Airliner. Journal of Aircraft Vol. 55, No. 4, 1509-1521 (2018).

21. Destarac, D. Far-Field / Near-Field Drag Balance and Applications of Drag Extraction in CFD, In: CFD-based Aircraft Drag Prediction and Reduction. VKI Lecture Series 2003, Von Karman Institute for Fluid Dynamics, Rhode Saint GenÃlse (February 3-7, 2003). 